\title{
ANÁLISIS DE LA EFICIENCIA ENERGÉTICA DE UNA RED DE ABASTECIMIENTO DE AGUA DE RIEGO A PRESIÓN ATENDIENDO A LA NATURALEZA DE LAS CONDUCCIONES.
}

\author{
Delgado de Molina, J. ${ }^{1}$, Martinez, F. $^{2}$, Bórnez, F. ${ }^{3}$, Bas, J. ${ }^{4}$
}

1 Ingeniero Agrónomo, Profesor T.U. Universidad Politécnica de Valencia. Departamento ingeniería cartográfica, fotogrametría, y geodesia, jdelgado@cgf.upv.es

2 Ingeniero Agrónomo, Jefe de obras y proyectos, Indefa Ingenieros, S.L.; Andarella 2, Esc. 2, 4º Pta. 14, 46950 Xirivella (Valencia), fmartinez@indefa.com

${ }^{3}$ Ingeniero Técnico Agrícola, Técnico, Indefa Ingenieros, S.L.; Andarella 2, Esc. 2, $4^{\circ}$, Pta. 14, 46950 Xirivella (Valencia), fbornez@indefa.com

${ }^{4}$ Ingeniero Agrónomo, Técnico, Indefa Ingenieros, S.L.; Andarella 2, Esc. 2, 4º, Pta. 14, 46950 Xirivella (Valencia), jbas@indefa.com

\section{Resumen.}

En la mayor parte de instalaciones de riego comunitarias modernizadas, que utilizan conducciones a presión para facilitar el manejo y la distribución del agua, los costes energéticos constituyen uno de los principales costes para los regantes. Se pretende con este trabajo, cuantificar los costes de energía disipada por fricción para una red de riego, en función del material adoptado para sus conducciones, poniendo de manifiesto las diferencias entre ellos. Con objeto de considerar la eficiencia energética, como un factor más a tener en cuenta a la hora de seleccionar uno u otro material, para el diseño de nuevas instalaciones. Para analizar el comportamiento de cada material, se ha establecido una comparativa entre ellos, mediante la utilización del modelo matemático de una red existente en la realidad, a través de un software de simulación hidráulica. La red estudiada ha sido la red de transporte de la Real Acequia de Escalona; la cual discurre íntegramente en término municipal de Villanueva de Castellón (Valencia), con una longitud total de $11 \mathrm{~km}$ de conducciones de Poliéster reforzado con fibra de vidrio (PRFV), con diámetros nominales comprendidos entre los $400 \mathrm{~mm}$ y los $800 \mathrm{~mm}$ y que se encuentra en funcionamiento desde el mes de junio de 2011. Los otros materiales estudiados han sido: Policloruro de vinilo orientado (PVC-O), Polietileno (PE), Acero (AC) y Fundición dúctil (FD). Tras el análisis realizado se ha podido determinar que el material que tiene un funcionamiento más eficiente (a igualdad de DN para cada tramo) es el PRFV, con unas pérdidas por fricción de 52,0 MW·h/año. Para el resto de materiales, esta cantidad puede verse incrementada entre un $17,5 \%$ y un $86 \%$.

\section{Abstract.}

In most of the modernised irrigation infrastructures, using pressurized pipelines in order to improve management and distribution of water, energy costs become one of the main costs for users. This work evaluates the cost of frictional pressure loss in an irrigation network, depending on the chosen material for pipes, in order to state differences among the materials. The objective of this work is to consider energy efficiency as a factor to keep in mind when selecting the material for pipes, in designing new networks. In order to analyze the performance of each material, we have established a comparative study among them, using the mathematical model of a real network with an hydraulic simulation software. The studied network is the Transport Network of "Real Acequia de Escalona", which is set up in Villanueva de Castellón (Valencia), with a total length of $11 \mathrm{~km}$ of glass-fiber reinforced plastic (GFRP), with nominal diameters between 400 and $800 \mathrm{~mm}$. This network is 
operational since June 2011. The other studied materials are Polyvinyl chloride (PVC), polyethylene, steel and cast iron. As a conclusion, the study shows us that the most efficient material (for the same diameter) is GFRP, with frictional losses of $52,0 \mathrm{MW} \cdot \mathrm{h} / \mathrm{year}$. For the rest of the materials, this amount could be increased between $17,5 \%$ and $86 \%$.

\section{Introducción.}

La estructura del coste energético asociado a la explotación de una red de riego es compleja, interviniendo en ella multitud de factores. El presente trabajo se centra exclusivamente en los relativos a la energía disipada por fricción, entendida como el "peaje energético" que hay que pagar para poder suministrar el agua a los usuarios a través de la red. En la magnitud de este peaje, juega un papel decisivo el material y la naturaleza de las conducciones que integran la misma.

El parámetro estudiado para evaluar, en cada caso, la eficiencia energética de la red estudiada, es el coste medio anual de pérdidas de carga por hectárea irrigada.

\section{Descripción del tema objeto de la comunicación.}

\subsection{Eficiencia energética en la distribución.}

En lo relativo a la distribución del agua en una red de riego a presión, se pueden distinguir claramente cuatro etapas:

- Presurización del agua en cabecera de la red.

- Distribución a través de la red de tuberías.

- Suministro a parcela a través de los hidrantes multiusuario y acometidas particulares.

- Consumo final en el interior de parcela.

De las cuatro, las dos primeras resultan clave a la hora de evaluar el consumo energético, y especialmente el sobreconsumo susceptible de ser reducido mediante actuaciones de eficiencia. Por el objetivo final del presente estudio, nos vamos a centrar exclusivamente en la etapa de distribución del agua a través de las tuberías.

Una vez que el agua entra en la red de tuberías, comienza la etapa de distribución propiamente dicha, circulando a través de todas las conducciones, desde las grandes arterias principales hasta los últimos ramales de menor diámetro, para ser entregada a los regantes a través de las tomas a parcela.

A lo largo de este recorrido, son dos los aspectos a considerar desde una perspectiva energética:

- Las pérdidas de carga (energía) por fricción.

- Las fugas de agua.

Por buenas que sean las condiciones de renovación, mantenimiento y operación de una red, la existencia de fricción y de fugas siempre será inevitable.

La mejor herramienta para poder evaluar ambos tipos de pérdidas es la realización de una auditoría energética sobre la distribución de agua a través de la red.

En tuberías nuevas y bien dimensionadas a los caudales a transportar, la tasa de pérdidas de carga por longitud (pendiente hidráulica) suele estar en torno a los $3 \mathrm{mca} / \mathrm{km}$, o menos.

En lo referente a las fugas, hay que decir que través de una fuga no escapa sólo agua de la red, sino que junto con ésta, también lo hace la energía de presión que mantiene 
acumulada desde su entrada en cabecera, y que de este modo se libera al exterior (se echa a perder) sin uso ni utilidad algunos.

Las fugas a su vez, también influyen directamente en las pérdidas de carga, pues en la medida en que aumenta el caudal transportado para compensar el que se pierde por las fugas, aumentan las pérdidas de carga.

Un planteamiento para el estudio conceptualmente muy sencillo, es el que formula Cabrera y col. (2010. Figura 1, tomada del mismo autor), y que consiste en entender la red de tuberías no como un transporte de agua, sino en el amplio sentido del término, como un transporte de energía.

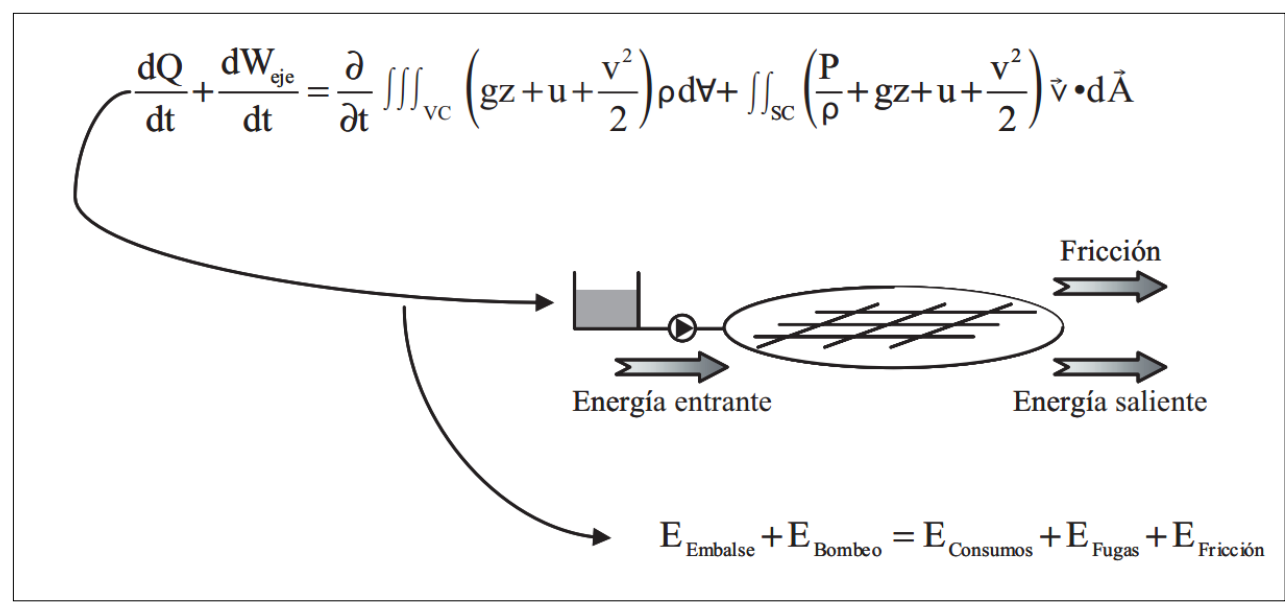

Figura 1. Planteamiento conceptual de la auditoría energética de una red y sus ecuaciones principales. Cabrera y col. (2010).

De forma paralela al agua que entra en la red por uno o más puntos (cabecera) y sale por otros (consumos y/o fugas), tal y como muestra la Figura 1, la red también tiene unas entradas y unas salidas de energía. Como entradas se encuentran:

- El aporte de energía potencial gravitatoria desde los depósitos de almacenamiento en cabecera.

- El aporte de energía elástica de presión por parte de los bombeos de apoyo que tenga.

Por otra parte las salidas de energía son las siguientes:

- El consumo de agua por parte de los regantes. Es decir, la energía de presión con la que se entrega el volumen a parcela a través de la toma individual.

- La fugas de agua, según se ha detallado más arriba.

La energía disipada por fricción. Este término, también detallado más arriba, no es una salida energética de la red en términos estrictos, pero sí supone, como disipación, un incremento de la energía contenida en el sistema

\subsection{Caso de estudio: Red de transporte de la Real Acequia de Escalona.}

La Red de Transporte de la Real Acequia de Escalona tiene una longitud de total de $11 \mathrm{~km}$ de tubería instalada. En naturaleza de poliéster reforzado con fibra de vidrio, presión nominal 10, rigidez nominal 5000 y rango de diámetros comprendidos entre 400 y $800 \mathrm{~mm}$. 
Esta red de transporte abastece a 7 cabezales, lleva en funcionamiento desde junio de 2011 y en la actualidad la superficie total irrigada es de 825,92 hectáreas repartida en aproximadamente 1900 tomas.

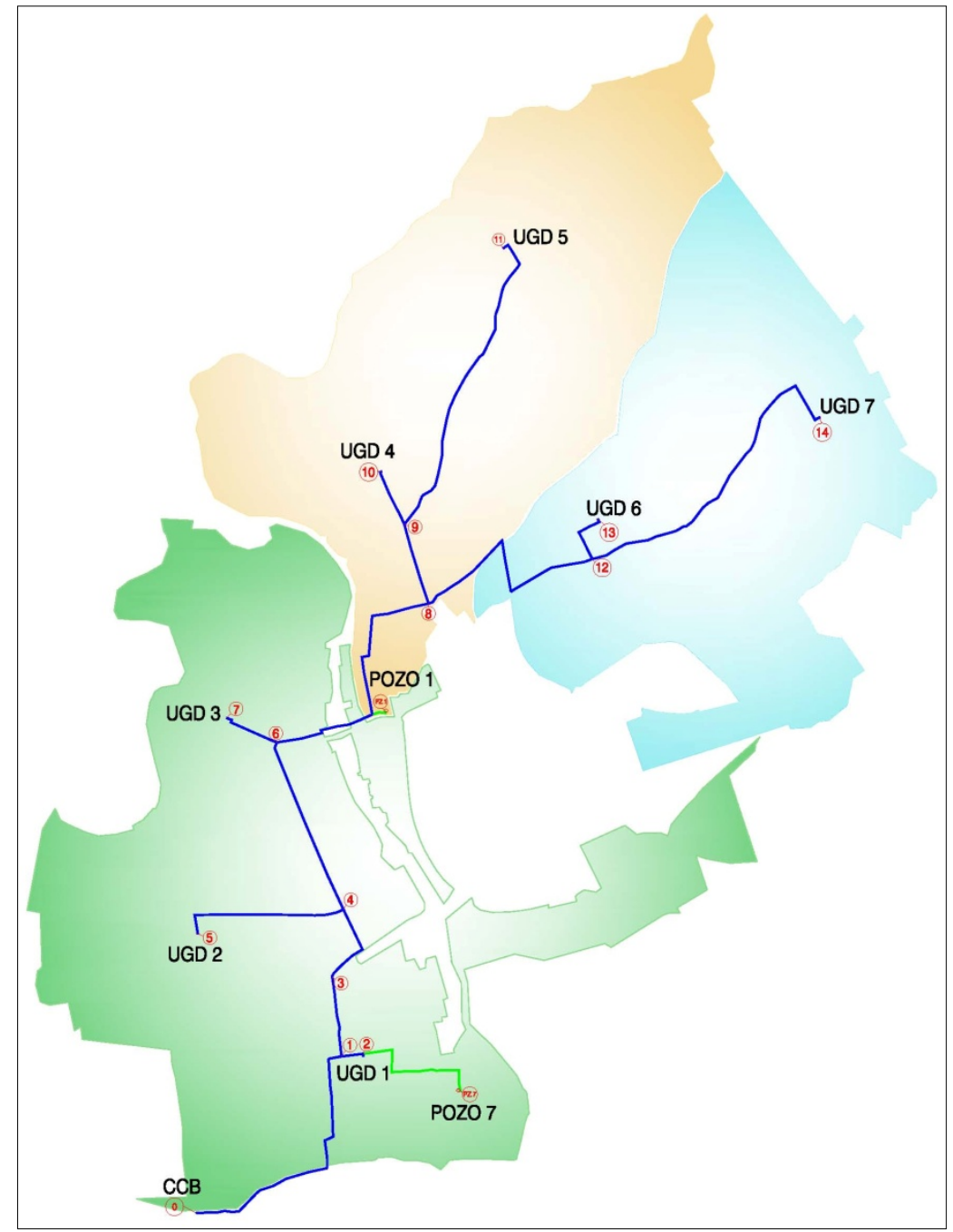

Figura 2. Superficie regable y red de transporte de la Real acequia de Escalona.

\subsection{Método de cálculo}

Se realizará el análisis hidráulico de la red con el programa informático EPANET. Se trata de una aplicación informática de difusión general desarrollada por la Agencia de Protección Medioambiental de Estados Unidos (US Environmental Protection Agency EPA). EPANET permite realizar simulaciones en periodo extendido del comportamiento hidráulico de redes de distribución a presión. Determina el caudal que circula por cada conducción y la presión en cada uno de los nudos en diferentes intervalos de tiempo. Entre sus múltiples funcionalidades calcula las pérdidas por fricción en las conducciones. Se ha utilizado la fórmula de Hazen-Williams para calcular las pérdidas por fricción. Los coeficientes adoptados para cada material han sido los siguientes: 
Tabla 1. Valores del coeficiente $C$ adoptado en el estudio según materiales

\subsection{Datos y topología de la red}

\begin{tabular}{|c|c|}
\hline Material & C \\
\hline Plásticos & 150 \\
\hline Fundición & 140 \\
\hline Acero & 120 \\
\hline
\end{tabular}

La red tiene su inicio aguas abajo de la estación de bombeo de captación directa del canal de la Real Acequia de Escalona. Desde este punto se transporta el agua a los 7 cabezales que distribuyen a toda la superficie de la comunidad de regantes. El riego de toda la superficie se realiza en 4 turnos de 2 horas de duración. Los parámetros básicos que configuran la simulación hidráulica son la presión en el inicio de la red y los caudales de demanda de cada cabezal. Estos datos están tomados del funcionamiento real de la red en la última campaña de riego. La presión en el inicio se ha tomado del transductor instalado aguas abajo de las bombas que nos indica la presión que proporciona la estación de bombeo para cada turno. Los caudales de demanda de los cabezales se han tomado de los datos de consumo registrados para cada cabezal. Obtenemos de esta manera 4 escenarios (4 turnos o intervalos de tiempo) para los cuales se realizará la simulación.

Tabla 2. Puntos de funcionamiento de la red.

\begin{tabular}{|c|c|c|}
\hline Turno & $\mathbf{Q}\left(\mathbf{m}^{\mathbf{3}} / \mathbf{h}\right)$ & $\mathbf{H}$ (mca) \\
\hline 1 & 2.062 & 44,68 \\
\hline 2 & 2.136 & 43,77 \\
\hline 3 & 2.071 & 44,58 \\
\hline 4 & 2.260 & 42,05 \\
\hline
\end{tabular}

En cuanto la topología, se trata de una red ramificada, con un dimensionado de diámetros comprendidos entre 400 y $800 \mathrm{~mm}$, con la siguiente distribución de longitudes

Tabla 3. Dimensionado de la red.

\begin{tabular}{|c|c|c|c|c|c|c|}
\hline DN $(\mathbf{m m})$ & $\mathbf{8 0 0}$ & $\mathbf{7 0 0}$ & $\mathbf{6 0 0}$ & $\mathbf{5 0 0}$ & $\mathbf{4 0 0}$ & Total \\
\hline Longitud (m) & 1.416 & 797 & 2.178 & 1.579 & 5.037 & $\mathbf{1 1 . 0 0 7}$ \\
\hline
\end{tabular}

En la siguiente tabla se indican los datos de longitudes y diámetros de la red:

Tabla 4. Datos de la red.

\begin{tabular}{|c|c|c|c|c|c|}
\hline Línea & $\begin{array}{c}\text { Nudo } \\
\text { inicial }\end{array}$ & $\begin{array}{c}\text { Nudo } \\
\text { final }\end{array}$ & $\begin{array}{c}\text { Cota nudo } \\
\text { final }\end{array}$ & $\begin{array}{c}\text { Longitud } \\
\text { (m) }\end{array}$ & $\begin{array}{c}\text { Diámetro } \\
\text { nominal } \\
\text { (mm) }\end{array}$ \\
\hline 1 & 0 & 1 & 44,18 & 1,00 & 800 \\
\hline 2 & 1 & 2 & 37,69 & $1.416,10$ & 800 \\
\hline 3 & 2 & 3 & 38,27 & 116,00 & 400 \\
\hline
\end{tabular}


XXXIII Congreso Nacional de Riegos

Universitat Politècnica de València, Valencia 2015

DOI:http://dx.doi.org/10.4995/CNRiegos.2015.1464

\begin{tabular}{|c|c|c|c|c|c|}
\hline Línea & $\begin{array}{c}\text { Nudo } \\
\text { inicial }\end{array}$ & $\begin{array}{c}\text { Nudo } \\
\text { final }\end{array}$ & $\begin{array}{c}\text { Cota nudo } \\
\text { final }\end{array}$ & $\begin{array}{c}\text { Longitud } \\
\text { (m) }\end{array}$ & $\begin{array}{c}\text { Diámetro } \\
\text { nominal } \\
\text { (mm) }\end{array}$ \\
\hline 4 & 3 & $\mathrm{C}-1$ & 38,46 & 37,50 & 400 \\
\hline 6 & 2 & 6 & 32,76 & 796,50 & 700 \\
\hline 7 & 6 & $\mathrm{C}-2$ & 33,68 & 856,80 & 400 \\
\hline 8 & 6 & 8 & 28,73 & 869,00 & 600 \\
\hline 9 & 8 & $\mathrm{C}-3$ & 26,85 & 328,40 & 400 \\
\hline 10 & 8 & 10 & 29,19 & 544,00 & 600 \\
\hline 12 & 10 & 12 & 27,74 & 764,90 & 600 \\
\hline 13 & 12 & 13 & 26,57 & 426,00 & 500 \\
\hline 14 & 13 & $\mathrm{C}-4$ & 27,31 & 276,00 & 400 \\
\hline 15 & 13 & $\mathrm{C}-5$ & 25,2 & $1.532,90$ & 400 \\
\hline 16 & 12 & 16 & 24,98 & $1.153,40$ & 500 \\
\hline 17 & 16 & $\mathrm{C}-6$ & 24,66 & 281,90 & 400 \\
\hline 18 & 16 & $\mathrm{C}-7$ & 22,69 & $1.607,80$ & 400 \\
\hline
\end{tabular}

La siguiente figura muestra un croquis de la red: 


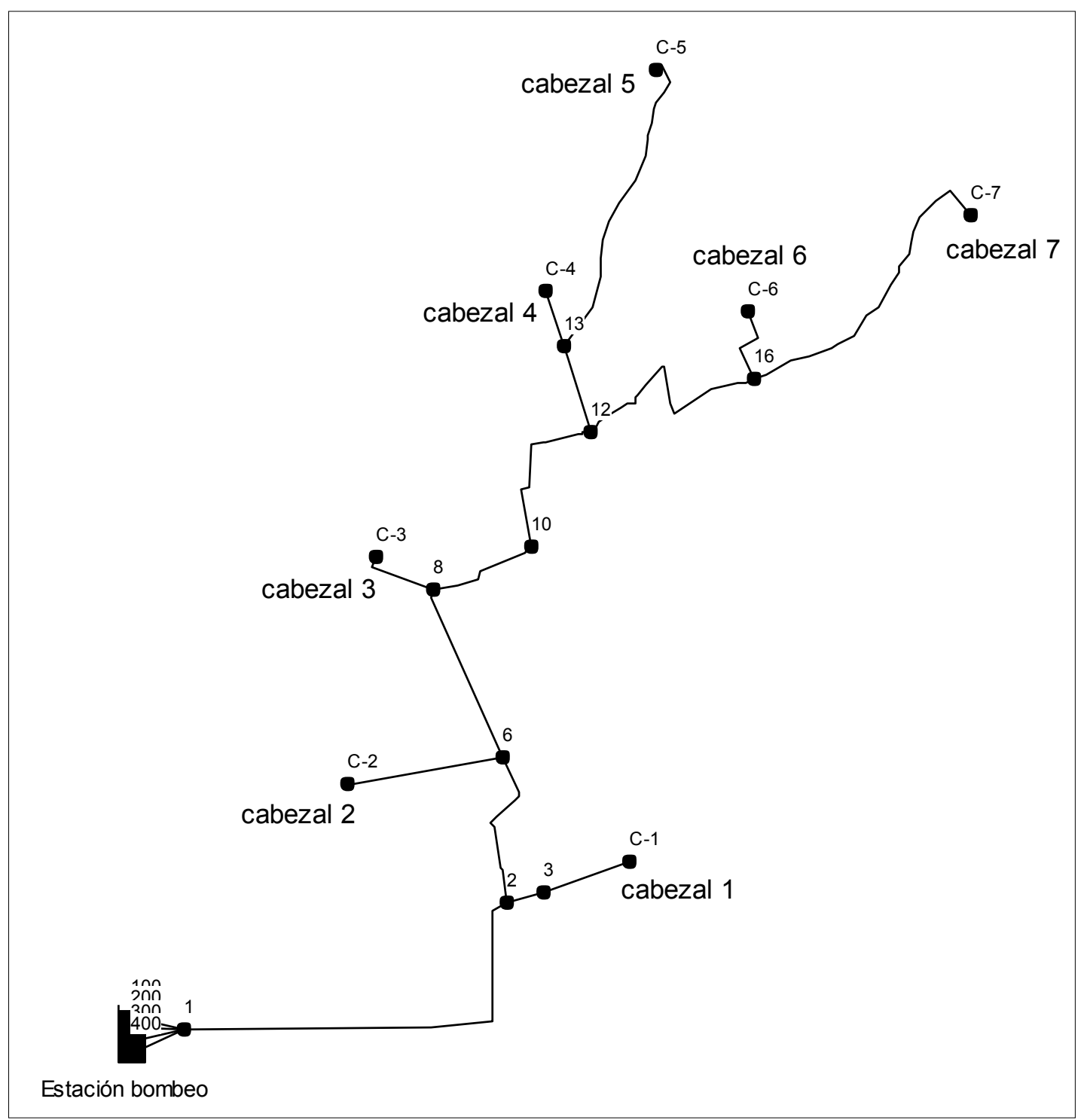

Figura 3. Topología de la red.

\subsection{Simulación hidráulica.}

A partir de los datos anteriores se ha realizado la simulación hidráulica del comportamiento de la red de transporte de la RAE para cada uno de los materiales que se incluyen en el análisis comparativo para este rango de diámetros, Poliéster reforzado con fibra de vidrio (PRFV), Policloruro de vinilo orientado (PVC-O), Polietileno (PE), Acero (AC) y Fundición dúctil (FD).

En el modelo de simulación hidráulica se han introducido las siguientes simplificaciones con la realidad.

1) Se ha considerado la situación ideal de ausencia total de fugas.

2) Se han eliminado del modelo las válvulas reductoras de presión que protegen los cabezales de riego de cada Unidad de Gestión Diaria (UGD), así como las de inicio de red (previstas para la conexión a las balsas de cota alta). 
3) Se han eliminado del modelo los pozos de sequía, pues están previstos para funcionar exclusivamente en situaciones de emergencia, en caso de que los recursos superficiales no resultaran suficientes.

Como resultados obtenidos de la simulación con EPANET para cada uno de los materiales se obtienen las presiones de servicio en los cabezales y los parámetros hidráulicos de las líneas (caudal, velocidad y pérdida de carga unitaria).

\subsection{Volumen de agua consumido.}

Se dispone del consumo de agua para el año hidrológico 2013 - 2014 (del 01/10/2013 al 30/09/2014) obtenido a partir de los datos de facturación facilitados por la Comunidad de regantes. El consumo total durante el año hidrológico ha sido de 2.983.778 $\mathrm{m}^{3}$.

\subsection{Superficie regada.}

La superficie de riego de la Comunidad de regantes a fecha 30/09/2014 era de $9.911,38$ hg $(825,92$ ha), según datos de la propia Comunidad.

\subsection{Consumos de energía en la Estación de bombeo RAE.}

El consumo energético total en la Estación de bombeo durante el año hidrológico 2013 - 2014, obtenido a partir de las facturas de la compañía suministradora desde el 01/10/2013 hasta el 30/09/2014 fue de 618.875,50 $\mathrm{kW} \cdot \mathrm{h}$. El importe total satisfecho a la compañía suministradora (impuestos incluidos), durante el mismo periodo fue de $76.776,17$ $€$. El coste medio del $\mathrm{kW} \cdot \mathrm{h}$ en el periodo estudiado fue de $0,124058 € / \mathrm{kW} \cdot \mathrm{h}$.

\subsection{Balances de energía}

El modelo de riego empleado consiste en abastecer la totalidad de la superficie en 4 turnos, con una duración de riego por turno de 2 horas. Los meses de máximas necesidades, se programa riego todos los días de la semana (7 riegos/semana). Los meses de menores necesidades se eliminan días de riego, pero no se modifica la duración de los mismos.

Por tanto el estudio realizado se particulariza para un riego y sus conclusiones serán extrapolables al total de riegos anuales.

En los balances de energía se ha despreciado en todos los casos el término cinético de la ecuación de Bernouilli o altura de velocidad, que para el rango de velocidades de la red adquiere valores inferiores a los $0,14 \mathrm{mca}$, lo que representa un $0,4 \%$ de la presión en los puntos de consumo.

Como hemos expuesto anteriormente, se considera la situación ideal de que no existen fugas, si tenemos en cuenta además que en la red objeto de estudio, no existe ningún punto elevado de acumulación (el agua se capta directamente de un canal en la que el agua entra por gravedad hasta la altura del eje de las bombas), la energía que entra en el sistema proviene íntegramente de la estación de bombeo, la cual se corresponde directamente con la entregada a los usuarios más las pérdidas por fricción.

$$
\mathrm{E}_{\text {Bombeo }}=\mathrm{E}_{\text {Consumos }}+\mathrm{E}_{\text {Fricción }}
$$


Como resultado de los balances de energía para cada uno de los materiales, se obtiene la energía total anual perdida por fricción para cada uno de ellos.

\subsection{Resumen final pérdidas de energía por fricción para cada material.}

Los resultados obtenidos en los balances anteriores para el plazo temporal de un año completo se recogen de forma resumida en la siguiente tabla:

Tabla 5. Pérdidas de energía por fricción para cada material.

\begin{tabular}{|l|c|c|c|}
\hline \multicolumn{1}{|c|}{ MATERIAL } & $\mathbf{E}_{\mathbf{f}}(\mathbf{k W} \cdot \mathbf{h} / \mathbf{a n ̃ o})$ & $\mathbf{E}_{\mathbf{f}} / \mathbf{E}(\%)$ & $\boldsymbol{\Delta}_{\mathbf{f}}(\boldsymbol{\%} \mathbf{P R F V})$ \\
\hline PRFV & $51.956,82$ & 14,61 & - \\
\hline PVC-O & $61.025,05$ & 17,16 & 17,45 \\
\hline Fundición & $66.639,70$ & 18,73 & 28,26 \\
\hline PE & $88.473,44$ & 24,87 & 70,28 \\
\hline Acero & $96.652,08$ & 27,17 & 86,02 \\
\hline
\end{tabular}

$E_{f}=$ Es la energía perdida por fricción en kWh/año y es distinta para cada material estudiado.

$E_{f} / E$ = Es la relación entre la energía inyectada a la red y la energía perdida por fricción en la misma en \% para cada material estudiado.

$\Delta \mathrm{E}_{\mathrm{f}}=\mathrm{Es}$ el incremento de energía perdida por fricción en \% de cada material estudiado, con respecto al más eficiente de todos (PRFV).

\section{Conclusiones.}

La energía inyectada en la red (E) es de 355,7 MW'h/año, aportada como hemos dicho anteriormente por el bombeo. Entre un 14,61\% y un 27,17\% (en función del material estudiado) se disipa por fricción.

Tal y como se puede ver en la tabla anterior, el material que tiene un funcionamiento más eficiente (a igualdad de DN para cada tramo) es el PRFV, con unas pérdidas por fricción de 52,0 MW/h/año, frente a las 61,0 MW·h/año del PVC, 66,6 MW·h/año de la fundición, 88,5 MW·h/año del PE y 96,7 MW·h/año del acero.

Comparativamente, se puede afirmar a partir de los datos obtenidos que el PVC presenta unas pérdidas por fricción un $17,45 \%$ superiores al PRFV, la fundición un 28,26\%, el PE un $70,28 \%$, elevándose hasta el $86,02 \%$ para el caso del acero.

Pero para poder inyectar esa cantidad de energía en la red $\mathrm{E}$, la estación de bombeo consume una cantidad de energía de la red eléctrica mucho mayor. Esa energía total, a la que denominamos $E_{b}$ es la queda registrada en el contador de energía eléctrica de la compañía suministradora y que para el periodo estudiado (01/10/2013 a 30/09/2014) es de

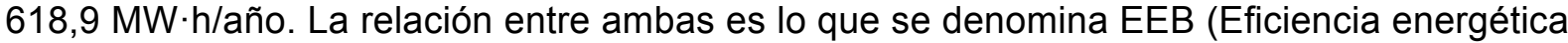
en los bombeos), y en nuestro caso es del $57,5 \%$ correspondiéndose con una Eficiencia Normal (Abadia y col. 2008).

La diferencia entre ambas es la energía total que se pierde antes de ser inyectada a la red y se corresponde con las pérdidas por rendimiento hidráulico de la instalación entre el punto de medida de la presión y la salida de las bombas $\left(\eta_{p}\right)$, rendimiento en las bombas $\left(\eta_{b}\right)$, pérdidas por rendimiento en los motores $\left(\eta_{m}\right)$, pérdidas por rendimiento en el cableado 
y aparellaje de maniobra entre el transformador y el motor $\left(\eta_{c}\right)$, pérdidas por rendimiento en variador de velocidad que acciona el motor $\left(\eta_{\mathrm{v}}\right)$ y pérdidas por rendimiento en el transformador $\left(\eta_{\mathrm{t}}\right)$.

$$
\text { EEB }=\eta_{p} \cdot \eta_{b} \cdot \eta_{m} \cdot \eta_{c} \cdot \eta_{v} \cdot \eta_{t}=\eta_{T}
$$

La relación entre la energía perdida por fricción $\left(E_{f}\right)$ y la energía empleada en el bombeo para vencer las pérdidas por fricción $\left(E_{b f}\right.$ medida en el contador de la compañía suministradora de energía) es la misma.

Por otra parte, si tenemos en cuenta que en función del uso que se hace de la energía en la estación de bombeo de la RAE $(94,9 \%$ en periodo "valle", $3,5 \%$ en periodo "llano" y $1,6 \%$ en periodo "punta"), el precio medio del $\mathrm{kW} \cdot \mathrm{h}$ (incluyendo término de potencia e impuestos) satisfecho a la compañía suministradora ha sido de 0,124058 €/ kW.h, se pueden establecer las siguientes relaciones:

Tabla 6. Energía empleada en el bombeo para vencer las pérdidas de energía por fricción para cada material.

\begin{tabular}{|c|c|c|c|c|c|}
\hline MATERIAL & $\begin{array}{c}E_{b f} \\
(k W \cdot h) / a n ̃ o\end{array}$ & $\underset{(€) / a n ̃ o}{E_{b f}}$ & $\begin{array}{c}E_{b f} \\
(k W \cdot h) / m^{3}\end{array}$ & $\begin{array}{c}E_{b f} / S \\
(k W \cdot h / h a) / a n ̃ o\end{array}$ & 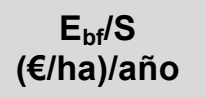 \\
\hline PRFV & $90.395,85$ & $11.214,33$ & 0,030 & 109,45 & 13,58 \\
\hline PVC-O & $106.172,99$ & $13.171,61$ & 0,036 & 128,55 & 15,95 \\
\hline Fundición & $115.941,51$ & $14.383,47$ & 0,039 & 140,38 & 17,42 \\
\hline $\mathrm{PE}$ & $153.928,43$ & $19.096,05$ & 0,052 & 186,37 & 23,12 \\
\hline Acero & $168.157,83$ & $20.861,32$ & 0,056 & 203,60 & 25,26 \\
\hline
\end{tabular}

Lo que supone un coste anual de $11.214,33 €$ en pérdidas por fricción en el transporte para el caso del PRFV. En el caso del PVC-O esta cantidad se incrementa en $1.957,26 €$, en el de la fundición en $3.169,14 €$, en el del PE en $7.881,82 €$, y en $9.647,00 €$ en el caso del acero.

Teniendo en cuenta que el volumen anual distribuido por la red de transporte es de $2.985 .150 \mathrm{~m}^{3}$ y que la superficie total irrigada es de 825,92 ha, podemos decir que los costes de energía por fricción soportados en la fase de transporte van desde los 13,58 €/ha para una red de PRVF, hasta los $25,26 € /$ ha de esta misma red confeccionada en acero. En el caso del PVC-O los costes son de 15,95 €/ha, de 17,42 €/ha para la fundición y de 23,12 $€ /$ ha para el PE. Para conocer exactamente el coste medio anual de pérdidas de carga por hectárea irrigada en cada caso, tendríamos que añadir a los costes anteriores, los costes de energía perdida por fricción en la fase de distribución, su obtención escapa del ámbito del presente estudio.

\section{Recomendaciones.}

Sería cuanto menos interesante, a tenor de los resultados obtenidos en el presente trabajo, el considerar la eficiencia energética, como un factor más a tener en cuenta, a parte de los tradicionalmente considerados (coste de adquisición y coste de instalación) a la hora de seleccionar uno u otro tipo de material, para el diseño de nuevas instalaciones. 


\section{Agradecimientos.}

Llegados a este punto, hemos de agradecer de forma especial la colaboración desinteresada recibida de la Real Acequia de Escalona, en particular de su personal técnico y administrativo, así como de su Junta Directiva. También queremos agradecer el apoyo prestado por la división técnica de la empresa Amiantit Spain S.A., sin el cual este trabajo nunca hubiese visto la luz.

\section{Bibliografía.}

- Arviza, J. y Santamarina, C., (1995), Ingeniería rural: Hidráulica. Ed. Servicio de publicaciones. UPV. Valencia.

- Montalvo, T., (2003), Ingeniería hidráulica. Ed. Inter Técnica Ediciones. Valencia.

- Rocamora, M. ${ }^{a}$ C.; Abadía, R.; Cámara, J.M.; Melián, M. ${ }^{a}$ A.; Puerto, H.; Ruiz, A., (2010), Manual de auditorías energéticas en comunidades de regantes.

- Uche, J., (2013). La energía en el agua. Ed. Barcelona

- Varios autores, (2003) Guía Técnica sobre tuberías para el transporte de agua a presión. CEDEX: Sección de Edición.

- Varios autores, (2012). Guía sobre Hidroeficiencia Energética. Comunidad de Madrid. 